\title{
STUDIES OF K LINE FILTERGRAMS
}

\author{
H. ZIRIN
}

Big Bear Solar Observatory, Hale Observatories, Carnegie Institution of Washington, California Institute of Technology, Pasadena, Calif. 91109, U.S.A.

(Received 27 May, 1974)

Abstract. High resolution filtergrams made with a $0.3 \AA \mathrm{K}$ line filter at Big Bear are discussed. The dark K 3 clouds noted in spectra by Bappu and Sivaraman (1971) are confirmed; they cover about $\frac{1}{3}$ of the inside of supergranule cells, and oscillate (mainly horizontally) with 3-4 min period. Their vertical extent of about $3000 \mathrm{~km}$ produces a sharp broadening of $\mathrm{K} 3$ at the extreme limb and obscuration of bright features.

Spicules also produce K 3 absorption, but they occur in emission more often than in $\mathbf{H} \alpha$, particularly near the limb. Apart from these differences the structures seen in $\mathrm{H} \alpha$ and $\mathrm{K}$ generally correspond.

A fine structure of granule size, also oscillating with 3-4 min period, is seen in $\mathrm{K} 1$. This structure is also obscured near the extreme limb, possibly by the same clouds seen in $\mathrm{K} 1$.

The bright K232 network does not appear to oscillate significantly in brightness, except through covering and uncovering by the overlying dark clouds. Some bright spicules, and many dark ones, are seen over all elements of the $\mathrm{K}$ network, as in $\mathrm{H} \alpha$.

In active regions the structure in $\mathrm{K}$ is very close to $\mathrm{H} \alpha$; all plagettes and plages are bounded by systems of strongly inclined bright and dark fibrils apparently marking the lines of force. The fibrils are the same (bright or dark) in $\mathrm{H} \alpha$ and $\mathrm{K} 3$; they are not seen in $\mathrm{K} 2$.

Umbral fiashes are much less visible in $\mathbf{H} \alpha$. There is, however, a finer, granular umbral structure in $\mathrm{H} \alpha$ which does not oscillate. Running penumbral waves are not seen in $\mathrm{K}$. The penumbra and surrounding areas are bright in $\mathrm{K} 3$. Every bright point in $\mathrm{K} 3$ corresponds to a magnetic field spot, except for the intranetwork area, where the magnetograms cannot detect possible fields. Flares are the same in $\mathrm{K}$ and $\mathrm{H} \alpha$.

The formation of the $\mathrm{K}$ double reversal at the limb is exhaustively discussed.

\section{Introduction}

Although the first spectroheliograms were made in the $\mathrm{K}$-line, and much of the early work was done in that line, the development of excellent Lyot filters for $\mathrm{H} \alpha$ has drawn chromospheric research toward interpretation of high resolution pictures in that line, so that, until recently (except for Beckers and Tallant's (1969) work on umbral flashes), there was little improvement over the beautiful $\mathrm{Ca} \mathrm{K}$ spectroheliograms made by Leighton and his coworkers with the Mt. Wilson 60-ft tower (Title, 1968; Zirin, 1966, pp. 228-232). No simultaneous $\mathrm{K}: \mathrm{H} \alpha$ pairs have appeared. Further, K-line movies with high resolution have not been previously obtained. The purpose of this paper is to display material obtained by operation of a Lyot filter for $\mathrm{K}$ at the Big Bear Solar Observatory since 1971 , to compare this material with simultaneous $\mathrm{H} \alpha$ observations, and to use the $\mathrm{K}$-line filtergrams to interpret the $\mathrm{K}$-line double reversal and the structure of the chromosphere in general.

The general appearance of K-line high resolution filtergrams may be summarized as follows:

In the wings there is a fine bright network which is obscured within $20^{\prime \prime}$ of the limb and a fine granular structure covering the Sun, oscillating with 3-4 min period. In the 
$\mathrm{K} 2$ peaks there is bright reversal principally confined to the edges of the network and not obscured at the limb. In K3 there is a sharp increase in absorption by rapidly oscillating dark clouds inside the cells, as well as by dark spicules (longer than in $\mathrm{H} \alpha$ ) near the limb. The K3 network is obscured, as is the K1 network, within $25^{\prime \prime}$ of the limb, but the sharpest effect is in the last $10^{\prime \prime}$. The clouds appear about $5^{\prime \prime}(3500 \mathrm{~km})$ high and about $10000 \mathrm{~km}$ across; they are presumably the general chromosphere.

There is a general correspondence between $\mathrm{H} \alpha$ and $\mathrm{K}$, except that:

(1) The contrast in $\mathrm{K}$, as is well known, is greater.

(2) Vertical bright features in $\mathrm{K}$ are more prominent.

(3) The spicules are darker in $\mathrm{H} \alpha$.

(4) The penumbra is bright in $K$.

(5) The $\mathrm{K}$ dark clouds are only seen in $\mathrm{H} \alpha$ centerline at the extreme limb.

Otherwise, the pictures in $\mathrm{H} \alpha$ and $\mathrm{K}$ are identical.

From our data we reach the following conclusions:

(1) The $\mathrm{K} 2$ reversal is predominantly a non-oscillatory brightness increase at the edges of the network cells,

(2) The K3 reversal consists mainly of rapidly moving dark clouds inside the cells, also visible (but less apparent) in $\mathrm{K} 1$ and 2 . Spicules also contribute to the $\mathrm{K} 3$ reversal.

(3) The K3 clouds represent the cool, general chromosphere inside the network cells. Since they only cover $\frac{1}{2}$ of the surface, they are not prominent in the disk center, but close to the limb their vertical extent $\left(\sim 5^{\prime \prime}\right)$ becomes important. Their horizontal velocity is higher than the vertical velocity, but this is not important until we get close to the limb and they dominate the absorption.

\section{Observational Procedure}

Lyot filters for the $\mathrm{K}$-line have been around for some time and have been operated by several observatories on a patrol basis. The reason high resolution pictures are difficult is quickly apparent when one sets the instrument up. Because of the limitations of polaroids in this spectral range, the filters built by Halle, which are the only ones available, use polarizing beam splitters, which produce an unwanted ordinary ray deviating about $5^{\circ}$ from the extraordinary ray, which is properly filtered. So the filter has to be far enough from the camera (about $30 \mathrm{~cm}$ at the least) for the beam to be blocked out. In addition, there are some internal reflections in the filter, due to the ordinary beam hitting the edges of the crystal elements, which must also be removed. After this is done, when one looks into the camera eyepiece to focus, the image is practically invisible because of the low sensitivity of the eye at that wavelength. All these factors make good $\mathrm{K}$ pictures difficult. Beckers suggests (private communication) that te polarizing crystals introduce some astigmatism. I have not tried to measure the astigmatism of the Halle filter at Big Bear, but examination of the transverse fibrils in our pictures shows them to be equally well focused in all orientations. It is also possible (Vrabec, private communication) that the seeing is worse in the K-line. 
The Halle K-line filter at Big Bear is operated in an $\mathrm{f} / 50$ beam on the 10 -in. refractor. Movies are made with $0.3,0.6$, and $1.2 \AA$ bandpass, depending on the program and the light level. Since $0.3 \AA$ is too wide to separate the $K$ peaks, one would prefer a narrower filter, but we don't have one at present. The exposures are in the order of $0.05 \mathrm{~s}$ on $\mathrm{S} 0392$, which is reasonably sensitive at this wavelength. Since the K line widens toward the limb and the K2 peaks are about $0.3 \AA$ apart in the disk center, the exposures near the limb tend to show more K3. Every two hours a ' $\lambda$ scan' across the line is made from -0.6 to $+0.6 \AA$.

\section{The K Line in the Quiet Sun}

The overall appearance of the chromosphere in $\mathrm{K}$ is well known from the medium resolution spectroheliograms already published by Leighton and co-workers. Since these have narrower passband than the filter, they can be used as a baseline to understand what we are seeing. In $\mathrm{K} 3$ there is a dominant bright network with a fairly dark area inside the cells; in $\mathrm{K} 2 \mathrm{v}$ and $\mathrm{r}$ we see bright points inside the cells, particularly in the former. In $\mathrm{Kl}$ the network is much weaker and its elements are narrower but bright points are very obvious inside the network cells. On films these are seen to oscillate in position and brightness with the $180 \mathrm{~s}$ period first measured by Orrall (1966). But in K3 the small features (all are less than $1^{\prime \prime}$ in diam) are much less apparent) in fact on filtergrams they are not seen at all near the limb where the band is more purely $\mathrm{K} 3$.

The filtergrams show more or less the same as spectroheliograms with higher spatial resolution and lower spectral resolution. The high resolution and the cine effect allows us to distinguish the features responsible for dark and bright parts of the line. Because the brightness at any point in the line is strongly time dependent (usually with about $240 \mathrm{~s}$ period) the bright and dark structures are easily identified as they wave about.

Figure 1 shows an $\mathrm{H} \alpha: \mathrm{K} 3$ pair at the extreme limb. We see that there is considerable difference between the two, mostly in contrast. At 1 we see a bright spicule in $\mathrm{K}$ which is much less prominent in $\mathrm{H} \alpha$. At 2 we see a dark cloud apparently connected with a spicule bush directly below it, but at 3 we see a cloud which is simply inside a cell. Even when we can't resolve spicules in $K$, in a movie it is easy to distinguish the two dark components by the fact that the spicules mostly shoot up and down while the dark clouds oscillate rapidly. In Figure 2, I try to show what is very clear in cine; two frames taken $140 \mathrm{~s}$ apart, at 200105 and 200325 (they are numbered to to correspond to Figure 1). At 4 we see a region which becomes dark at 2003, while the area just to the right, which was dark, is now light. Other changes are apparent at 5 and 6 , the latter being a brightening presumably due to uncovering. Whenever the dark clouds fade, we may see through to the underlying fine structure of the upper photosphere. The reader will easily find other changes in this short interval; on cine projection a violent oscillation is seen. Note that the spicules are relatively unchanged.

The correspondence between $\mathrm{H} \alpha$ and $\mathrm{K}$ is still very good; the dark feature 2 is seen 


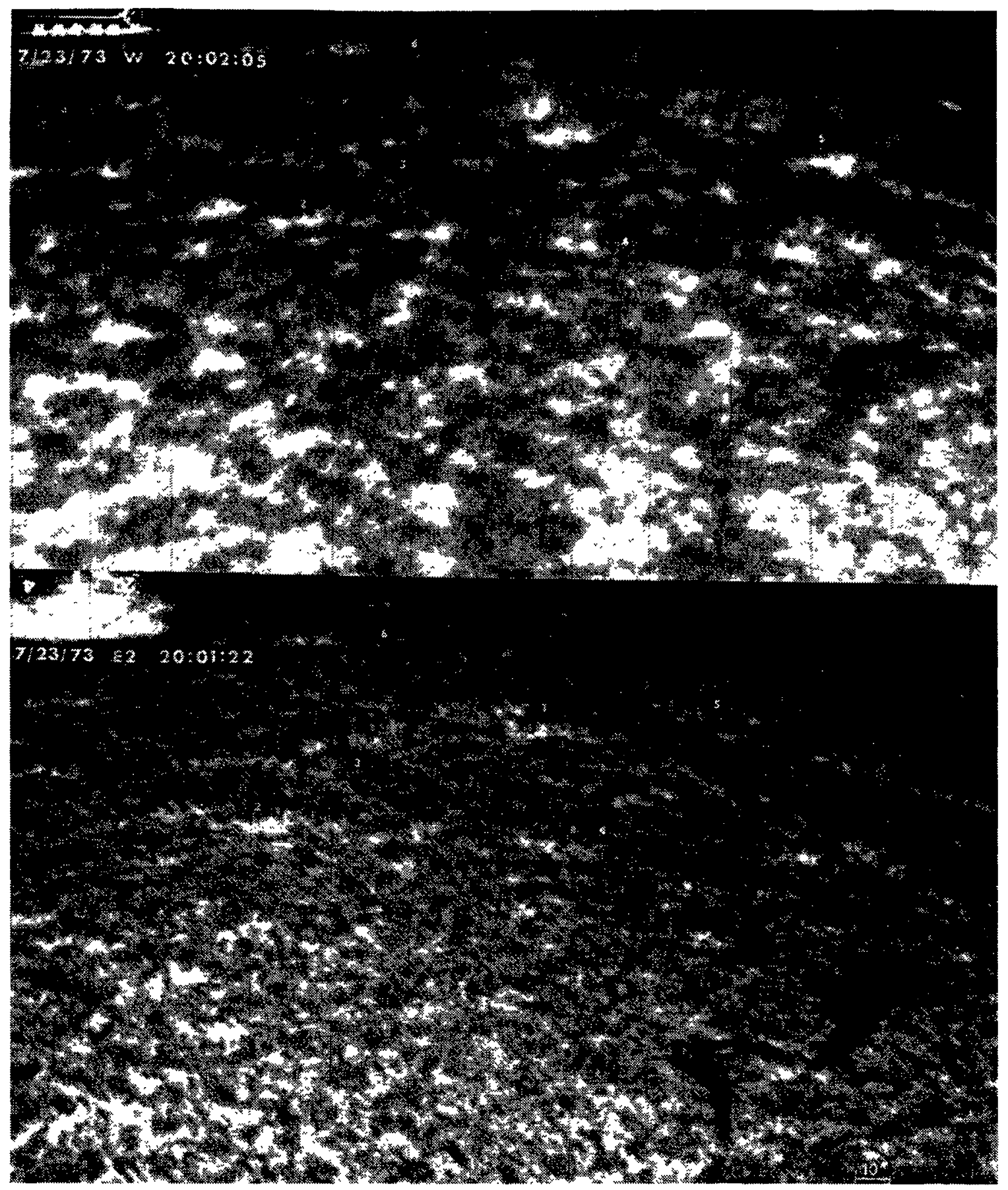

Fig. 1. Close frames in $\mathrm{K} 3$ (top) and $\mathrm{H} \alpha$ (bottom) of the north pole of the Sun. Corresponding features are numbered and described in the text. Note especially the bright and dark spicules at 1 and the many dark clouds near the limb.

on the $\mathrm{H} \alpha$ frame to be resolved into a group of spicules, while the dark feature 3 is part of a horizontal intracellular structure. Plagettes like 1 are obviously larger and brighter than the others; in Figure 3, which shows the same scene at $-0.6 \AA$, we see that this plage, as well as some others, has longer and larger spicules, stronger emission and more complex structure. In $\mathrm{K}$ we see it has bright spicules. It probably corresponds to X-ray bright points reported by Vaiana et al. (1973). 


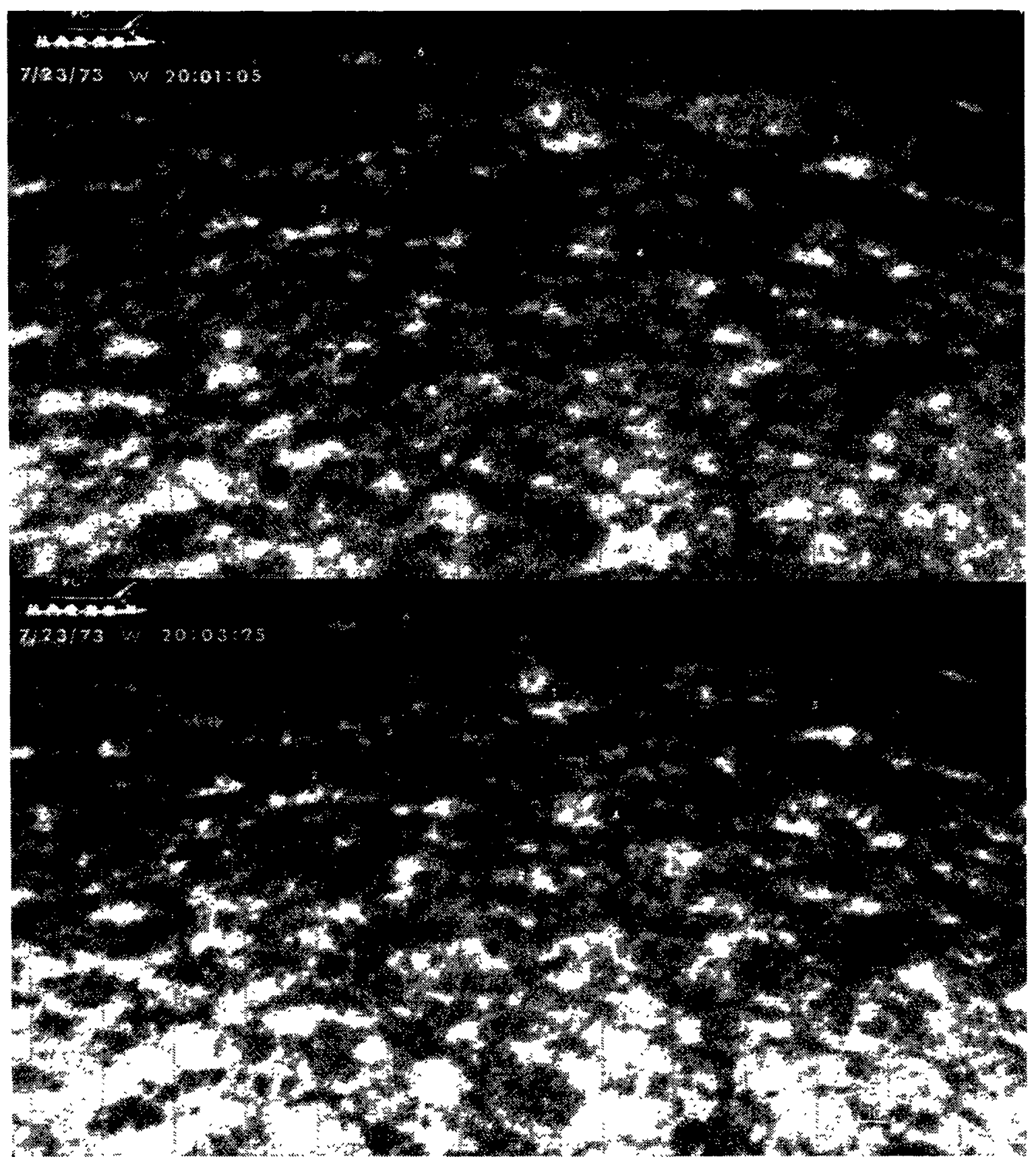

Fig. 2. Two frames, $140 \mathrm{~s}$ apart, before and after the preceding, which show the rapid changes in the dark absorbing clouds that make up the general chromosphere. Features are numbered to correspond with Figure 1.

The dark clouds seen in K3 (whicin were originally pointed out by Bappu and Sivaraman (1971)) appear to move predominantly horizontally, which accounts for the fact that large excursions in $\mathrm{K} 3$ are not seen in the center of the disk. The resolution of our films is such that vertical oscillatory motions of a few arc seconds would be easily seen.

To better understand the $\mathrm{K}$-line, one must examine a wavelength scan such as is shown in Figure 4. Frames taken July 22 at $-0.3,0.0,+0.3$ and $-0.6 \AA$ are shown. Although the resolution of the filter is not what we would like, the peaks are clearly 


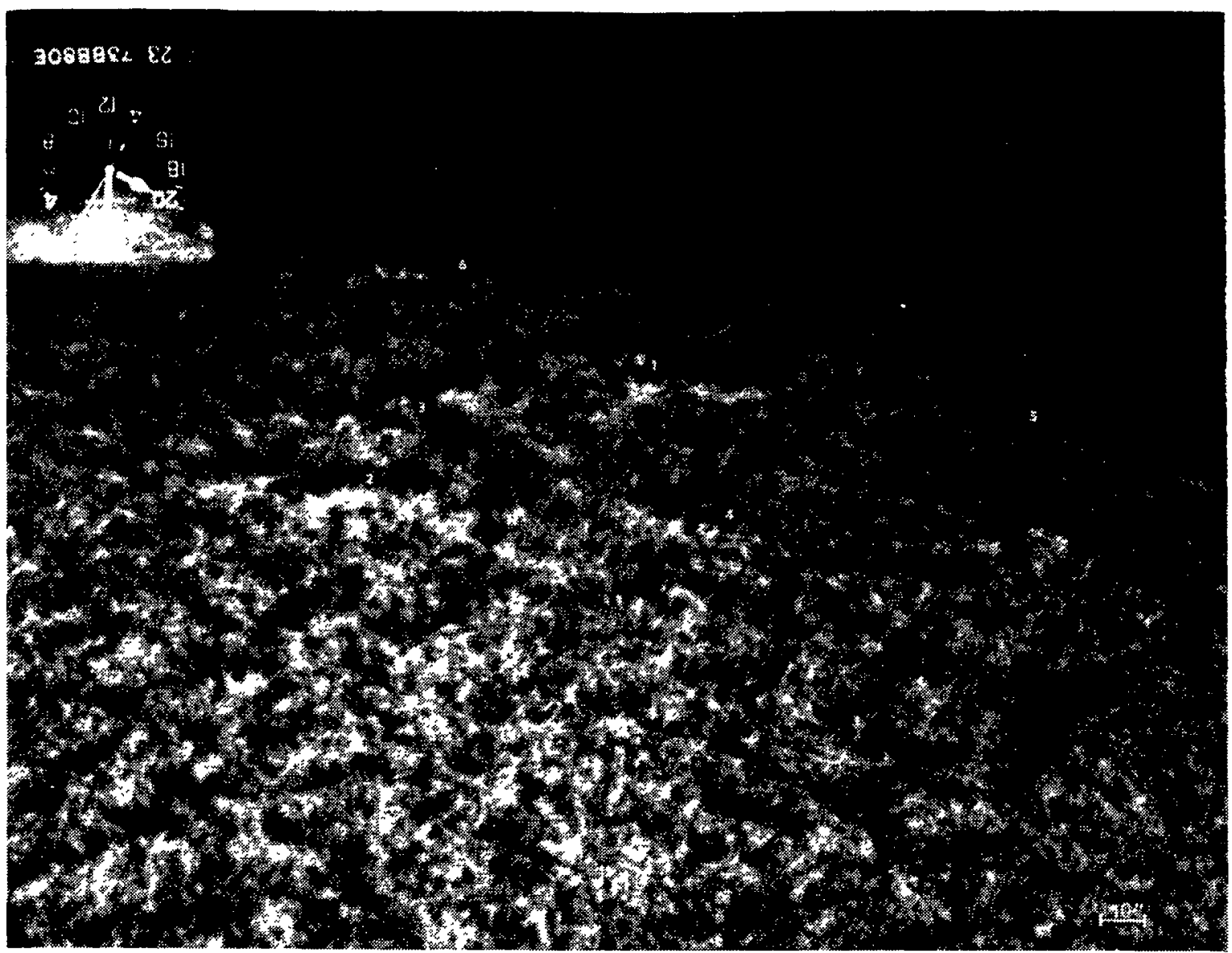

Fig. 3. An excellent $\mathrm{H} \alpha-0.25 \AA$ frame on July 23, 20:01:05. Compare with Figure 2a. This shows very well the structure seen in $\mathrm{K} 1$. But the dark clouds cannot be seen, although they occasionally appear on movies at this wave length. The bright plagettes correspond well.

separated. In particular we may see the double reversal at the limb; the bright point 7 is bright in $\mathrm{K} 2 \mathrm{v}$ and $\mathrm{r}$ but disappears completely in $\mathrm{K} 3$ and $\mathrm{K} 1$; on the other hand the plagette 8 appears both in $\mathrm{K} 2$ and $\mathrm{K} 3$. Examples of the double reversal at the extreme limb have appeared in various published spectra. The explanation appears to be that the emission is narrow enough so that it does not occur in $\mathrm{K} 1$, and is absorbed by the dark clouds in $\mathrm{K} 3$ which obscure the path to the limb. In the center of the disk there is no obscuration, so the $\mathrm{K} 23$ peaks in the network are flat, without $\mathrm{K} 3$ absorption, while at the limb there is a sharp $\mathrm{K} 3$ absorption in the network elements. This is clearly because the dark clouds have some vertical extent, and while they are limited to part of the inside of the cells at the disk center, they may obscure the network near the limb. So the observations fit the model in this respect.

The one effect that does not fit this model is the disappearance of the $\mathrm{K} 1$ structure near the limb. The $\mathrm{K} 3$ absorption must be narrow, or $\mathrm{K} 2$ emission near the limb would not be seen. Yet the same sort of structures can be seen obscuring the K1 network. We can only guess that the $\mathrm{K} 3$ absorbers have broad wings, which suffice to reduce the already low contrast in $\mathrm{K} 1$.

Liu (1974) has reported observation of a time lag between brightening in K1 and 


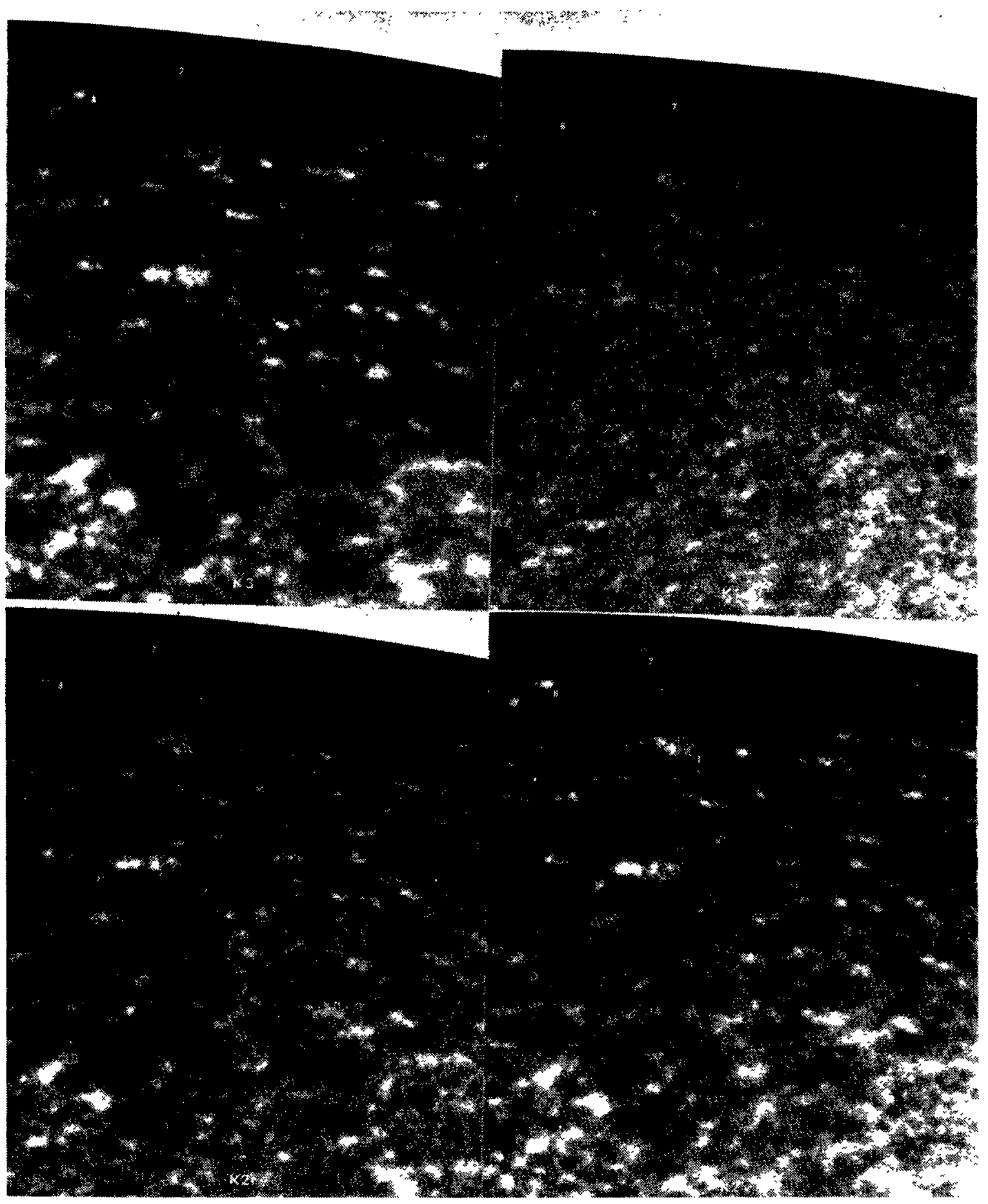

Fig. 4. A scan across the $\mathrm{K}$ line on the preceding day, July 22; wavelengths are marked; $\mathrm{K} 3$, 20:03:17; K1v (K3-0.6 $), 20: 01: 58 ; \mathrm{K} 2 \mathrm{v}(\mathrm{K} 3-0.3 \AA), 20: 03: 15 ; \mathrm{K} 2 \mathrm{r}(\mathrm{K} 3+0.3 \AA), 20: 03: 22$. The K1V frame has been chosen to test Liu's hypothesis that brightenings in $K 1$ are followed by brightenings in $\mathrm{K} 2 \mathrm{v}$ with $100 \mathrm{~s}$ lag. The reader will of course make his own comparisons, but I find that few of the brightenings in $\mathrm{K} 1 \mathrm{v}$ are connected with subsequent $\mathrm{K} 2$ brightenings. 
$\mathrm{K} 2 \mathrm{v}$ in various parts of the chromosphere. He does not give the location of these phenomena or the guiding stability of the telescope; from the spectra they appear to be plagettes. Our films are ideal for looking for this phenomenon, particularly when a K1 movie (made with $1.2 \AA$ bandpass) is followed by $\mathrm{K} 2$ or $\mathrm{K} 3$ (the filter is broad enough so either will do). No effect was found. It must be recalled that bright points in $\mathrm{K} 1$ corresponds to those in $\mathrm{K} 2$; although there is constant oscillation, the brightenings in $\mathrm{K} 2$ seem to be more closely connected with lateral motion and uncovering by moving the dark clouds than the wave from below beloved of theoreticians. Figure 4 shows a $\mathrm{Kl}: \mathrm{K} 2$ pair with 2 min delay; there is no special relation between the bright points other than the agreement of the network. As many are bright after as before. Further, since $K$ is known to show a 4 min period, Liu's 2 min lag of $K 2$ could just as well be a lead. Liu's spectra give an excellent opportunity to study the K-line; unfortunately only two are accompanied by spectroheliograms. These are in or near active regions, so they shed little light on the normal line. Further, a single frame is inadequate for quiet sun interpretation as the rapid oscillation makes successive frames have different appearances. On the other hand the present work is severely limited by our lack of spectra, and can only be viewed as a guide to phenomena which we shall study with spectroscopy.

It is important to realize that plages and network have about the same brightness in $\mathrm{K} 2$ and $\mathrm{K} 3$, but the intracellular region is much darker in $\mathrm{K} 3$. The reality of this effect which is a little hard to prove by comparing pictures, may be seen by examining the slow-scan spectroheliogram obtained by Leighton and published by Title (1966, No. 24) and by Zirin (1966, p. 228). In this picture one sees that the background changes very little between $\mathrm{K} 1$ and $\mathrm{K} 2$, and then darkens sharply in K3. In the filtergrams this darkening is seen to cover the $1^{\prime \prime}$ fine structure seen in $\mathrm{K} 1$, and mostly to consist of rapidly moving dark clouds which are very prominent near the limb. By contrast, the emission features that form the network and plages are brighter in K2 and 3 than in $\mathrm{K} 1$; thus there is a real difference in the source function gradient with height between the inside and outside of the cells.

In Figure 5 we present yet another $\mathrm{H} \alpha: \mathrm{K}$ pair, made in 1971. Although the $\mathrm{H} \alpha$ is probably the best we have ever made inside the limb, the K-line is made with only $0.6 \AA$ pass band, so it mixes $\mathrm{K} 2$ and 3 and shows the emission features as well as the low-lying small elements which are presumably connected with the granulation. A number of features have been marked on either print; the two are simultaneous with parallel 10" refractors. The following may be seen : 2,7, classical "bushes' (Cragg et al., (1963), the plagette visible in both, the spicules only visible in $\mathrm{H} \alpha$ (the absence in $\mathrm{K} 232$ is unimportant, as we know the spicules are best seen in $\mathrm{K} 3$ ); 3, 4 regions somewhat obscured in $\mathrm{H} \alpha$, but showing bright vertical spicules in $\mathrm{K} 232$; 6 , a region marked by long bright and dark spicules in $\mathrm{H} \alpha$, and some dark spicules in $\mathrm{K}: 8$, a complex region showing spicules both pointing toward the limb and coming towards us, appearing also in $\mathrm{K}$.

In this pair several dark clouds may also be seen in both lines, such as the small dark cloud just below 7 and the group left of 5 . 


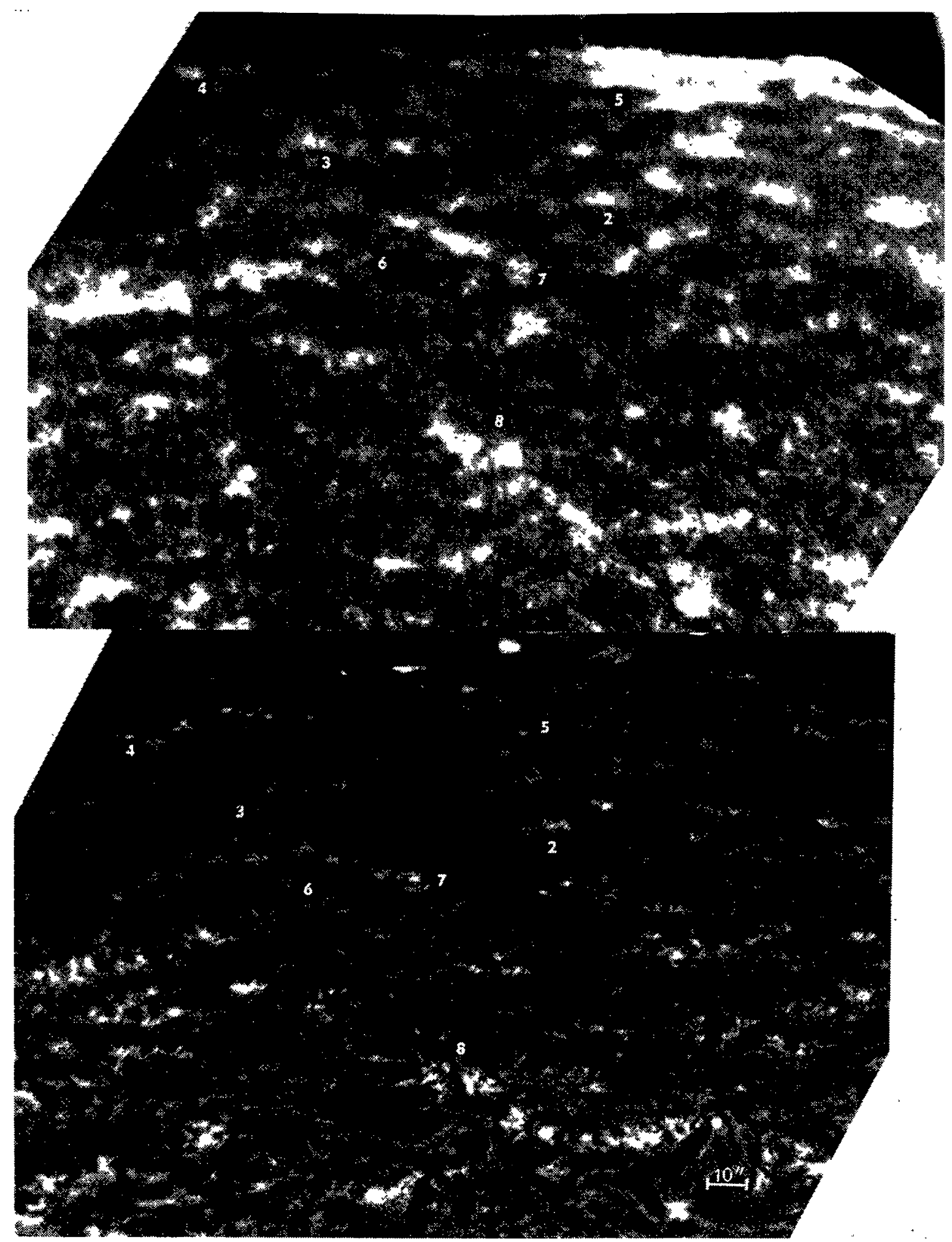

Fig. 5. An $\mathrm{H} \alpha$ : K pair taken September 23,1971 . The $\mathrm{K}$ line, top, is with $0.6 \AA$ bandpass, combining $\mathrm{K} 232$. The $\mathrm{H} \alpha$ pair below is one of the best made so far at Big Bear. The dark obscuring clouds of the general chromosphere are easily seen in $\mathrm{H} \alpha$ to have a mainly horizontal structure. Plagette 4 is obscured in $\mathbf{H} \alpha$ but shows vertical emission features in $\mathrm{K}$. 
Probably the dark clouds which absorb K3, and are barely seen in K232 here, are the clouds which produce the relative diminution in the $\mathrm{H} \alpha$ intensity toward the extreme limb. If we use the position of the K232 plagettes to determine where there might be spicule bushes, we see that there are simply not enough bushes. They are obscured by the dark clouds. This shows up not quite so well in Figure 1b. It is known that spicules above the limb are not easily seen in centerline, but one must go to $\pm 0.8 \AA$ in $\mathrm{H} \alpha$. The chromosphere seen in centerline is essentially composed of these clouds. Figure 5 shows what a $10^{\prime \prime}$ telescope can do with nearly perfect seeing and contrast; the elongated features greatly enhance the signal: noise ratio, and although most features are $1^{\prime \prime}$ across, many features only $0.25^{\prime \prime}$ across may be measured.

In conclusion, the $\mathrm{K} 3$ absorption may be seen to arise from several sources:

(1) A general diffuse absorption which cannot be defined on the filtergrams.

(2) Dark clouds $(l \sim 10000 \mathrm{~km})$ inside the network cells and covering about $\frac{1}{3}$ of their area. Presumably the chromospheric temperature inside the network cells continues to drop with no heating, giving rise to cool dark clouds, which, however, move very rapidly with $180-240$ s period.

(3) Spicules, coming up out of the edges of the network.

The $\mathrm{K} 2$ emission comes from the following:

(1) Plagettes, the bright regions on the edges of the network.

(2) Spicules.

(3) Small grains inside the network cells. These are much less bright than the first two.

All of these are much brighter in the violet, and we can shed no light on this problem.

$\mathrm{K} 1$ is seen to be dominated by a multitude of fine low-lying grains with strong oscillation.

The reader will have noted that the $\mathrm{K}$-line illustrations enable us to investigate day to day changes in the chromospheric network. This was done for three days, July 21, 22,23 . As the reader can also see by comparing Figures 1 and 4 , the detailed correspondence is lost overnight, although some plagettes such as 1 , maintain their identity. If we follow a continuous film for $12 \mathrm{hr}$, we find no particular discernible change, mostly because we can follow the evolution of each section. Individual frames are also misleading because the plagettes are covered and uncovered by dark clouds out of phase, so that the locus of a network element can only be established by watching a movie.

\section{The K-Line in Active Regions}

Most of our observations are in active regions, where we find the $\mathrm{K}$ observations mostly useful for umbral flashes and observing very weak fields. The high contrast in active regions is a drawback so far as lattuide is concerned, but it also enables a lower modulation transfer function to be used, giving higher resolution. Figures 6 and 7 show simultaneous $\mathrm{H} \alpha$ and $\mathrm{K}$ pictures in McM 12417, where we got particularly good data. We see that there is a good correspondence in the features. The intense plage 1 


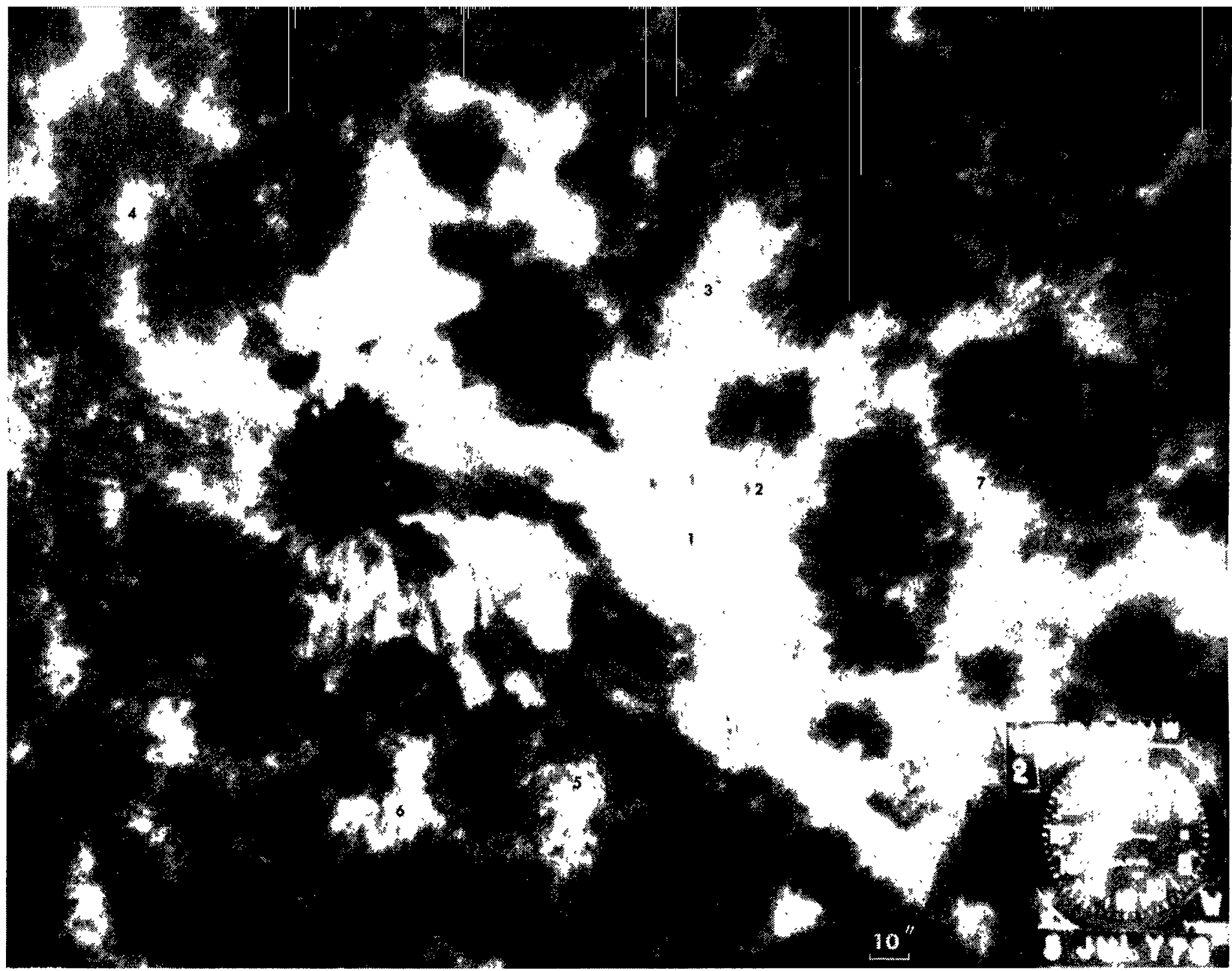

Fig. 6. A high resolution K3 frame of McMath 12417 taken 19:01:46 July 5, 1973. Various features explained in the text are enumerated. Umbral flashes inside the spot and bright and dark fibrils extending from the plage are particularly visible. 


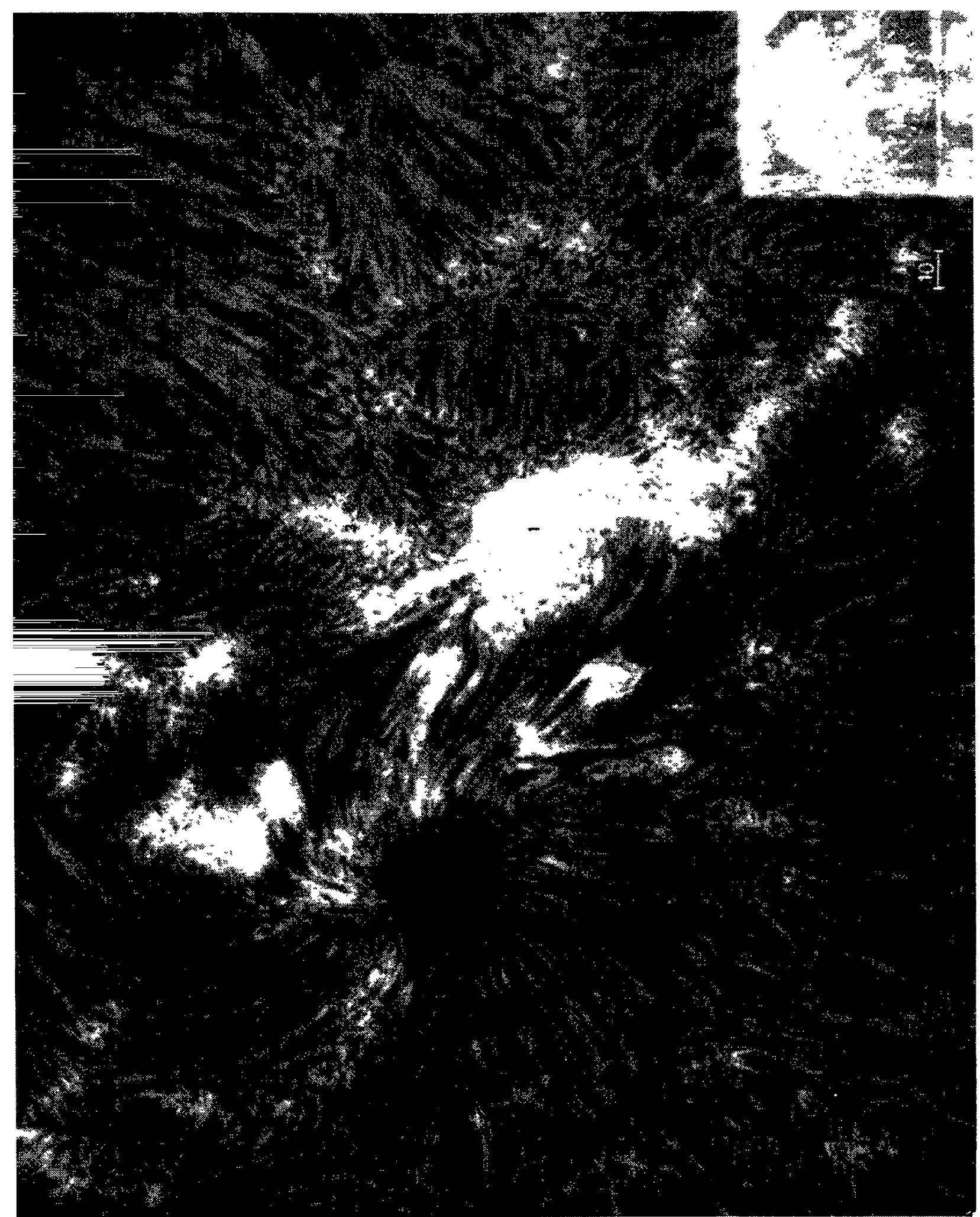


is bright in both $H \alpha$ and $K$. But the plage 2 is not bright in $H \alpha$, although some bright points appear. The $\mathrm{K}$ structure there is less bright than 1 and appears somewhat open, but is clearly bright. This area, which is bright in $\mathrm{K}$ and marked only by granular structure in $H \alpha$ centerline and dark structure off-band, we shall call the subplage. The subplage shows enhanced magnetic field on magnetograms, but not so much as in the ordinary plage; also the field is more broken and granular. The Skylab photos obtained by NRL (Tousey et al., 1973) show that coronal enhancements are limited to the plage, i.e. the $\mathrm{H} \alpha$ emission region. These results appear to be quite general; we find them in all cases. Surrounding the active region is what we have referred to as the enhanced network; the elements of this network, which were called plagettes, rosettes, or bushes by various authors, are strikingly similar to the subplage, but they may (such as 3 and 4) be closer to plage in brightness. Even a casual examination of these figures reveals both bright and dark fibrils. Careful examination of plagettes like 5 and 6 , shows that bright fibrils in $\mathrm{H} \alpha$ are also bright in $\mathrm{K}$, and dark fibrils also appear in both lines; this is true everywhere but in the penumbral area. Only in $\mathrm{K}$ the background is much darker so the dark fibrils are lost. But below the numeral 6 we easily can see such correspondence as well as to the right of 7 . The fact that the fibril struc-

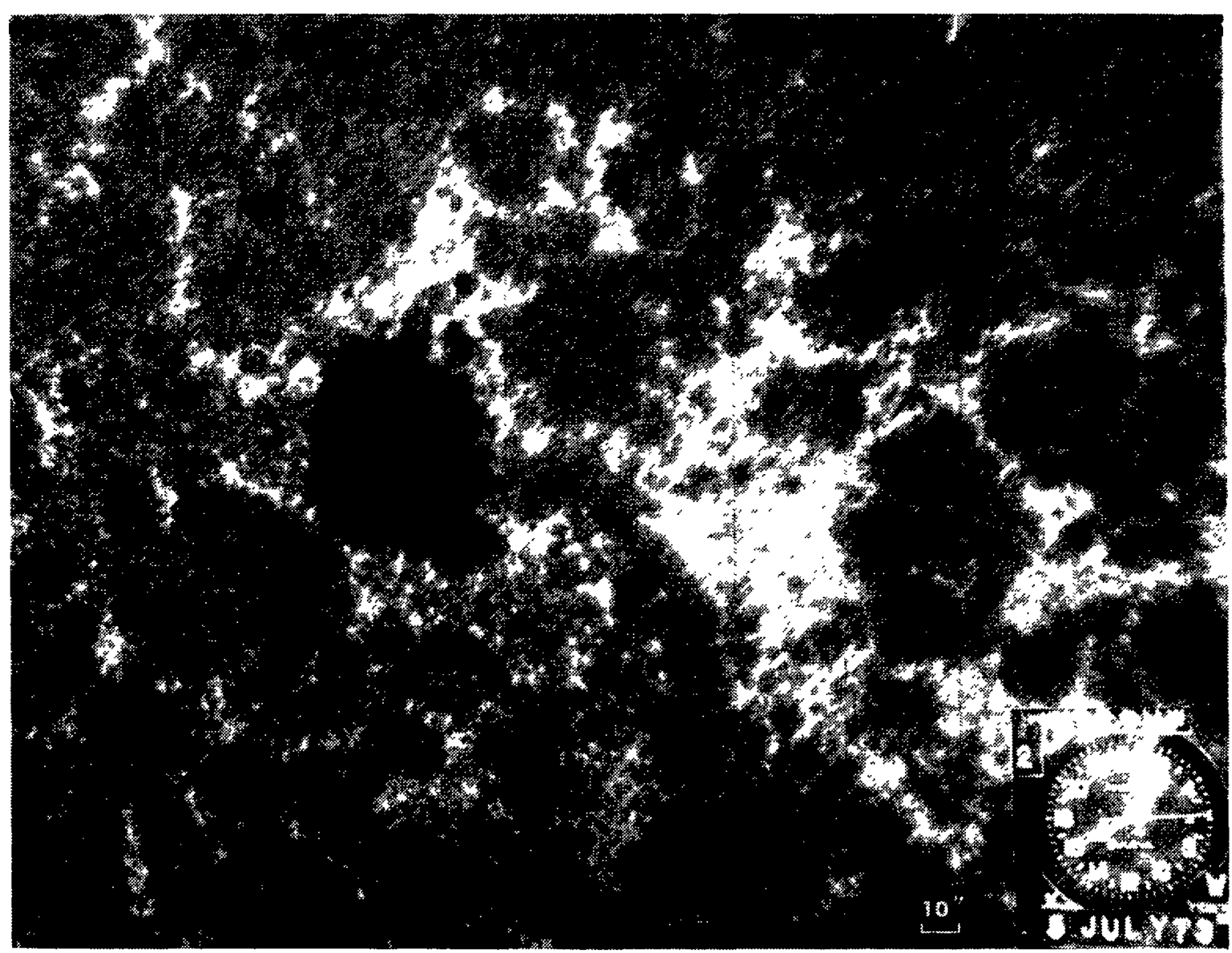

Fig. 8. The same at $0.6 \AA$ to the blue of the $\mathrm{K}$ line, showing detailed fine structure of granule size, presumably due to the photospheric granulation. Taken one hour before Figures 6 and 7 . 
ture in $\mathrm{K}$ is identical with that in $\mathrm{H} \alpha$ implies that important effects in the $\mathrm{K}$-line may be due, not to some peculiar effect of a homogeneous layer, but to the accidental juxtaposition of different structures along the line of sight. The reader should remember that our filter has a half-intensity width of but $0.3 \AA$, and the fibrils are much more prominent in spectroheliograms (Sheeley, 1971) with narrower bandpass. The occurrence or absence of a K3 reversal at any point will therefore depend only on the existence of overlying dark or bright fibrils from nearby plagettes. This of course is what we have referred to in the previous section on the quiet chromosphere near the limb. Figure 8 shows a print of the same region at $\mathrm{K} 3-0.6 \AA$, made an hour earlier. The $\mathrm{K} 1$ structure is much finer, essentially granule size, and corresponds to the $\mathrm{CN}$ network dicussed by Sheeley and the filigree discussed by Dunn and Zirker (1973).

The activity around sunspots divides itself into two classes, umbral flashes and penumbral activity. In the umbra, flashes were first detected by Beckers and Tallant (1969) and are easily seen in $\mathrm{K}$. There is a complex $\mathrm{H} \alpha$ fine structure in (or, more accurately, above) the umbra but it does not appear to correspond to the $\mathrm{K}$ flashes. On the other hand, we have clearly seen the umbral flashes in $\mathrm{H} \alpha$ in other sunspots, in particular McM 11856. We conclude that the umbral flashes are a higher structure, only occasionally visible in $\mathrm{H} \alpha$, and that there is a lower lying fine structure in the um-

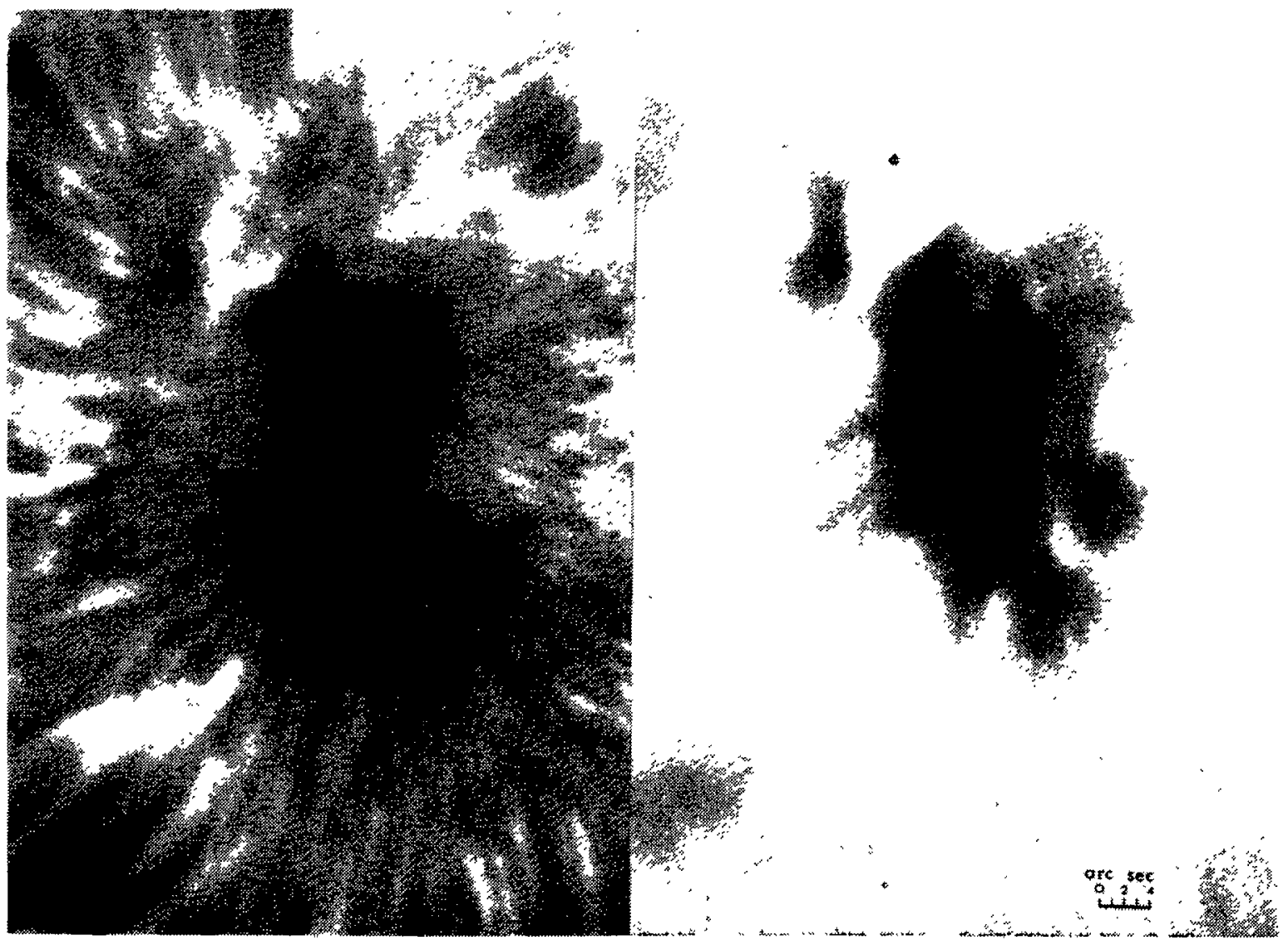

Fig. 9. Two simultaneous highly enlarged views of the umbra of McM 12417, $\mathrm{H} \alpha$, heavily dodged, at left; and $\mathrm{K} 3$, not dodged, at right. The $\mathrm{H} \alpha$ shows a background structure which does not oscillate much, while $\mathrm{K}$ shows bright oscillating structures. 
bra. Figure 9 show greatly enlarged simultaneous views of McM 12417 obtained July 2 ; note that the two are quite dissimilar. The $\mathrm{K}$ line (right) shows a coarse structure which oscillates rapidly. The $\mathrm{H} \alpha$ (left) shows a slowly changing fine structure fairly uniform across the spot. This must be connected with the umbral granulation. The $\mathrm{K}$ flashes are quite invisible at $\pm 0.3 \AA$ and must have a narrow profile; this is consonant with the well-known narrowing of the $\mathrm{K} 2$ emission to a single $\mathrm{K} 3$ peak. Its presence means there is matter above the spot with the same source function as that in the plage, but with much less optical depth. The fact that the running penumbral waves are not seen in $\mathrm{K}$ may have some height information, but the penumbral structure in $\mathrm{K}$ is so broken that these waves would be hard to detect, and besides, they are only visible with excellent resolution, which we do not quite have in $\mathrm{K}$.

We have not discussed the appearance of flares in $\mathrm{K}$ because that has been already covered by Zirin and Lazareff (1974). We can distinguish no significant difference between the two lines. However, there is often considerable activity around light bridges which is much more apparent in $\mathrm{K}$ because of the heightened contrast. In Figure 10 we show an interesting small flare which occurred around 1915 on July 5. It is seen in $\mathrm{H} \alpha$ and $\mathrm{K}$ as two bright footpoints, one inside the umbra, and one (marked by an arrow) in the following plage. Presumably the lines of force in which the energy was released loop above the region and connect the spot with the plage. Although this flare was superimposed on a strong umbral flash pattern, it did not oscillate, but died out to uncover the normal oscillation. This implies that either the flare represents a steady source of energy to the layer of $\tau=1$ in K3, or it dumps its energy in a higher layer in which oscillations do not take place. Even if the input were steady, any oscillation involving density change would tend to produce brightness changes. The absence of these favors the view that the umbral flashes are an excitation wave (presumably magnetosonic) producing brightness changes in existing irregularities.

We have not yet discussed the beautiful, complex structure around the umbra. Although similar in $\mathrm{H} \alpha$ and $\mathrm{K}$ so far as outline is concerned, it is obviously much brighter in K. Some of this effect is because the absorbing overlying dark fibrils seen in $\mathrm{H} \alpha$ (e.g. Figure 7) are more transparent in K, but most seems to be a real increase in $\mathrm{K}$ line source function in these regions. Since the opacity of penumbral matter is high in $\mathrm{K}$ relative to $\mathrm{H} \alpha$ (the penumbra is cool and $\mathrm{H} \alpha$ excitation is low). The area below the spot (Figure 8) was discussed by Roy and Michalitsanos (1974) as the locus of a wave of opposite polarity moving out from the sunspot.

It is interesting that each and every plagette seen in $\mathrm{H} \alpha$ and $\mathrm{K}$ has a set of fibrils radiating away from it in the direction of some opposite polarity, near or far. In active regions these have low inclination, since nearby fields pull them downward (this is easily seen at the limb), whereas in quiet regions they break the surface at higher angles. The length of these fibrils and spicules measures in each case the complex transition region to the corona. Beyond the ends of each fibril seen must lie coronal material. Presumably higher resolution will reveal the bright and dark fibrils to be different stages in this evolution. In cine the fibrils are seen to be continually changing, both by fading in and out and by shooting up and down. 

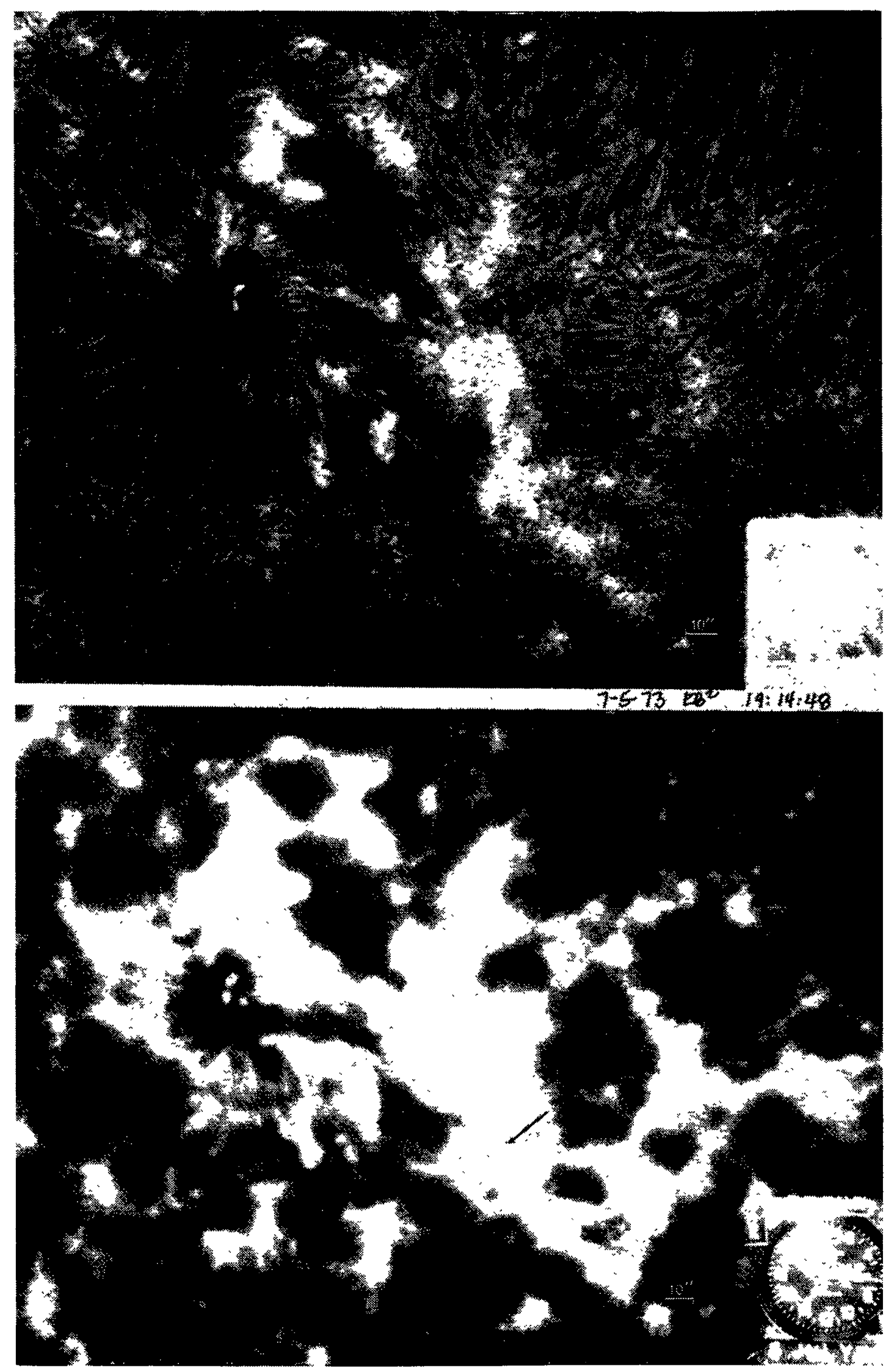

Fig. 10. A small flare on July 5, with one branch in the center of the umbra and the other, in the following plage (arrow). The two branches must be connected by magnetic arches across the middle of the group. 


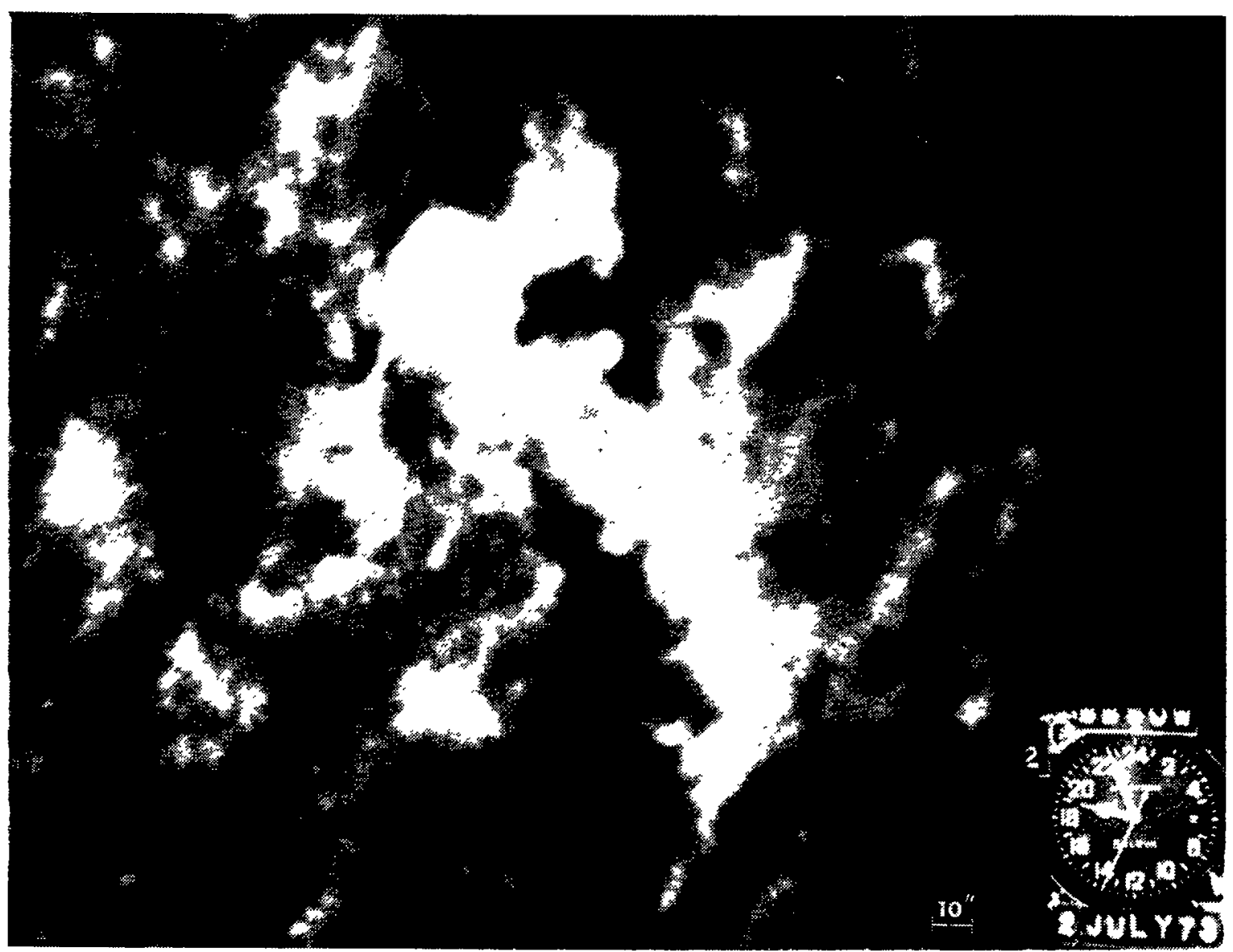

Fig. 11. A K line frame of the same group July 2, showing how little it changed. The group hardly changed from July 1 to 6 , on July 7, however, an emerging flux region came up in the middle, growing to a second $p$ spot and accompanied by many flares as it spread. The large spot has been separately overexposed to show the umbral flash structure; in a uniform $\mathrm{K}$-line print it would be dark, as in Figure 10.

Finally, Figure 11 shows the same spot group on July 2, three days earlier. Note that there was very little change in these three days, particularly in the close plage. The principal changes are in the perimeter at right where there has been outward expansion (but one must allow for fore-shortening), and in the region around the main spot (particularly below it) where the plages present July 2 are weakened or have disappeared. This is presumably due to the outflow of polarity observed by Roy and Michalitsanos; it is a means by which the sunspot grows; an $f: p$ pair surfaces in the spot, and the $f$ part flows out, cancelling nearby $p$ polarity and leaving a net increase of $p$ polarity in the spot. Thus this outflow in effect concentrates magnetic flux into the sunspot from its surroundings.

Finally we should note the perfect correspondence between the simultaneous magnetograms already published by Roy and Michalitsanos. It is possible that some of the weakest $\mathrm{K}$ features also correspond to magnetic fields too weak to be detected by magnetographs. 


\section{Summary}

We have discussed many features appearing on $\mathrm{K}$ line filtergrams. The chief difference from $\mathrm{H} \alpha$ is involved with the complexities of the double reversal; we see in $\mathrm{K} 3$ most clearly the absorption of the cool clouds of the general chromosphere, which cover about $\frac{1}{3}$ the area inside the cells, oscillate rapidly and have a vertical extent around $3000 \mathrm{~km}$. The confluence of these clouds in the line of sight accounts for the sharp increase in the separation of the $\mathrm{K} 2$ peaks at the extreme limb, as well as the obscuration of spicules in $\mathrm{H} \alpha$ centerline above the limb. The clouds also produce some effect in $\mathrm{K} 1$, indicating a small component with high velocity or broad wings.

In active regions the $K$ structure mimics $H \alpha$, except that the penumbra and related regions are bright in $\mathrm{K}$ and dark in $\mathrm{H} \alpha$. Bright and dark fibrils in both lines match up. The umbral flashes are much less apparent in $\mathrm{H} \alpha$ than in $\mathrm{K}$. Flares are the same.

\section{Acknowledgements}

I wish to thank Dr Ronald Moore for many stimulating discussions. This work was supported by NASA under NASA NGL 05002 034, the Atmospheric Sciences section of NSF under Ga. 24015 and by AFCRL under F19628-73C-0085.

\section{References}

Bappu, M. K. V. and Sivaraman, K. R.: 1971, Solar Phys. 17, 316.

Beckers, J. and Tallant, P.: 1969, Solar Phys. 7, 351.

Cragg, T., Howard, R. F., and Zirin, H.: 1963, Astrophys. J. 138, 303.

Dunn, R. B. and Zirker, J. B.: 1973, Solar Phys. 33, 281.

Liu, S.: 1972, Ph. D. Thesis, Univ. of Maryland.

Liu, S.: 1974, submitted to Astrophys. J.

Orrall, F. Q.: 1966, Astrophys. J. 143, 917.

Roy, J. R. and Michalitsanos, A.: 1974, Solar Phys., 35, 47.

Sheeley, N. R.: 1971, Solar Phys. 20, 19.

Title, A.: 1968, Selected Spectroheliograms, Caltech Bookstore, Pasadena, Calif.

Tousey, R., Bartoe, J.-D., Bohlin, J. D., Brueckner, G. E., Purcell, J. D., Scherrer, V. E., Sheeley,

N. R., Jr., Schumacher, R. J., and Vanhoosier, M. E.: 1973, Solar Phys. 33, 265.

Vaiana, G. et al.: 1973, Astrophys. J. 185, L47.

Zirin, H.: 1966, The Solar Atmosphere, Blaisdell, Waltham, Mass.

Zirin, H. and Lazareff, B.: 1974, Solar Phys., in press. 